\title{
New economical and simple device for intraoperative expansion on small and medium sized soft tissue defects
}

Jun Won Lee,

Seong Hoon Park,

Seong Joo Lee,

Seong Hwan Kim,

Hii Sun Jeong,

In Suck Suh

Department of Plastic and

Reconstructive Surgery, Kangnam

Sacred Heart Hospital, Hallym

University College of Medicine, Seoul, Korea
Intraoperative expansion has been used to cover small to large defects without disadvantages of the conventional tissue expanders. Various materials, for example, expanders and Foley catheters are being used. We introduce a new, convenient and economical device immediately available in the operating room, according to the defect size for intraoperative expansion, with latex gloves or balloons. The retrospective study was done with 20 patients who presented with skin and soft tissue defects. During the operation, expansion was done with latex gloves or balloons inflated with saline through an intravenous line and a three-way stopcock. After the inflation, the glove was removed and skin was covered with expanded tissue. A careful decision was made regarding the inflation volume and placement of the expander according to the defect size. There were no postoperative complications. The skin contracture and tension was minimal with a texture similar to the adjacent tissue. The new intraoperative expansion devices with latex gloves and balloons were cheap and made easily right in the operation room. The reconstruction of small to large sized skin defects can be done successfully, functionally and aesthetically without using expensive commercial materials.

Keywords: Tissue expansion / Surgical procedures, operative / Equipment and supplies

\section{INTRODUCTION}

Efforts are being made to improve results in the reconstruction of soft tissue defects, with minimal scarring. Surgical methods for defect restoration include primary suture, skin graft, local flap, tissue expansion and free flap. The simplest and most effective method has to be selected individually. Typically, a local flap is the first method to be considered as it ensures that the color, tone, sensitivity, thickness and epidermal appendages are maintained in the affected area. However, there are limitations

\footnotetext{
Correspondence: In Suck Suh

Department of Plastic and Reconstructive Surgery, Kangnam Sacred Heart

Hospital, Hallym University College of Medicine, 1 Singil-ro, Yeongdeungpo-gu,

Seoul 07441, Korea

E-mail: sismdps@chol.com

Received July 4, 2018 / Revised August 12, 2018 / Accepted August 22, 2018
}

and disadvantages of the local flap. It needs sufficient amount of tissue and the remaining scar can be unfavorable according to the shape of the flap and size of the defect. Thus, tissue expansion is widely used to allow more flexible application of local flaps and to achieve aesthetically and functionally desirable results which resemble that of the primary closure without tension.

Conventional tissue expansion often requires more than two surgeries which are costly and time-consuming. As the tissue expands over several weeks, body disfigurement is a major downside. However, intraoperative sustained limited expansion (ISLE), attempted during an operation by Sasaki [1] in 1987, shows good prognosis, expanding the skin beyond its original limit using excess tissue to restore up to $3-5 \mathrm{~cm}$ of soft tissue area without tension and distortion. This was the beginning of 
intraoperative expansion being recognized as a surgical method that can maximize the merit of tissue expansion while negating its weaknesses.

Intraoperative expansion yields fewer negative effects associated with conventional tissue expansion, such as several operative steps and temporary deformity resulting from the use of a tissue expander. Various materials, for example, expanders and the Foley catheters were being used. We now introduce a new, convenient, and economical device for intraoperative expansion using latex gloves or balloons.

\section{IDEA}

This retrospective study was conducted using data from 20 patients who presented with skin and soft tissue defects (Table 1). Mean age was 33 years (range, 5 to 65 years), and mean followup period was 11 months (range, 6 to 26 months). This study was approved by the Institutional Review Board of Kangnam Sacred Heart Hospital in Seoul, Korea (IRB No. 2016-09-118) and followed the principles of Declaration of Helsinki.

Key indications include (1) scars or defects of which widths are larger than $4 \mathrm{~cm}$ and difficult to perform primary closure; (2) inelastic donor sites such as scalp, which could experience complications from primary suturing of a small tissue defect; (3) anatomically important areas such as the lower eyelid, the commissura labiorum and the nasal alar area, which could experience distortion or displacement when the skin is closed un- der high tension.

Intraoperative expansion was performed using a latex glove inflated with saline through an intravenous line and a threeway stopcock (Fig. 1). While the patient was under general or local anesthesia, a central incision or elongation of the incisions of the pre-existing defects was made and the area to be expanded was undermined to place the expansion device in a pocket located below the subcutaneous tissue or above the periosteum. If the defect was too big to achieve satisfactory expansion with one device, two or three devices were inserted. The inflation of the expansion device was conducted through pushing normal saline at the stopcock. While the device was being inflated, the protrusion of the device beyond the pocket was avoided by pressing down on the area of entry with fingers. Tissue expansion was conducted by increasing the volume of the expanding device through repetitive inflations and deflations. Although there was variation between areas, the first inflation was typically 10-20 mL for 5 minutes, followed by 5 minutes of deflation, followed by a second inflation of $30-50 \mathrm{~mL}$ for another 5 minutes. The final volume was about $50-100 \mathrm{~mL}$, up to $400 \mathrm{~mL}$ in some cases. The volume was depending on the size and flexibility of defect which the defect can be closed with minimal tension. After the tissue was expanded sufficiently, the flaps were pulled together to test the tension, then the device was removed and the defect was primarily closed without tension. According to the defect size, a careful decision has to be made regarding the inflation volume (number of glove digits or bal-

Table 1. Clinical features of patients who had intraoperative expansion

\begin{tabular}{|c|c|c|c|c|c|c|c|}
\hline Location & Case no. & Age (yr) & Defect size $\left(\mathrm{cm}^{2}\right)$ & Cause & Materials ${ }^{\mathrm{a})}$ & Underlying disease & Complication \\
\hline \multirow[t]{6}{*}{ Scalp } & $1^{\text {b) }}$ & 24 & $8 \times 10$ & Trauma & Latex glove (1) & No & No \\
\hline & 2 & 48 & $3 \times 6$ & Trauma & Latex glove (2) & No & No \\
\hline & $3^{\text {b) }}$ & 7 & $4 \times 4$ & Nevus sebaceous & Balloon (2) & No & No \\
\hline & $4^{\text {b) }}$ & 45 & $5 \times 16$ & Burn & Balloon (3) & No & No \\
\hline & 5 & 50 & $5 \times 5$ & Soft tissue necrosis & Balloon (2) & DM & No \\
\hline & $6^{\text {b) }}$ & 5 & $3 \times 5$ & Nevus sebaceous & Latex glove (1) & No & No \\
\hline \multirow[t]{2}{*}{ Forehead } & $7^{\text {b) }}$ & 25 & $3 \times 4$ & Trauma & Latex glove (1) & No & No \\
\hline & $8^{\text {b) }}$ & 30 & $3 \times 3$ & Nevus & Latex glove (1) & No & No \\
\hline \multirow[t]{3}{*}{ Canthal area } & $9^{\text {b) }}$ & 35 & $3 \times 3$ & Nevus & Latex glove (1) & No & No \\
\hline & 10 & 60 & $2 \times 2$ & Trauma & Latex glove (1) & HTN & No \\
\hline & $11^{\text {b) }}$ & 38 & $2 \times 3$ & Scar & Latex glove (1) & No & No \\
\hline \multirow[t]{2}{*}{ Nose } & $12^{b)}$ & 34 & $2 \times 2$ & Trauma & Latex glove (1) & No & No \\
\hline & 13 & 65 & $3 \times 3$ & Trauma & Latex glove (1) & HTN, DM & No \\
\hline \multirow[t]{4}{*}{ Chin and lip } & 14 & 15 & $3 \times 5$ & Nevus & Latex glove (1) & No & No \\
\hline & 15 & 55 & $4 \times 4$ & Tumor & Latex glove (1) & No & No \\
\hline & $16^{\text {b) }}$ & 42 & $2 \times 3$ & Scar & Latex glove (1) & $\mathrm{DM}$ & No \\
\hline & 17 & 18 & $3 \times 3$ & Burn & Latex glove (1) & No & No \\
\hline \multirow[t]{3}{*}{ Cheek } & $18^{\text {b) }}$ & 20 & $4 \times 5$ & Nevus & Latex glove (1) & No & No \\
\hline & $19^{b)}$ & 35 & $3 \times 3$ & Tumor & Latex glove (1) & No & No \\
\hline & 20 & 12 & $3 \times 4$ & Burn & Latex glove (1) & No & No \\
\hline
\end{tabular}

DM, diabetes mellitus; HTN, hypertension.

${ }^{a}$ Number of balloons and digits: (1) indicates one digit of glove; (2) two digits of glove or two balloons; (3) three digits of glove or three balloons; ${ }^{\text {b)Follow-up period longer than } 6}$ months. 

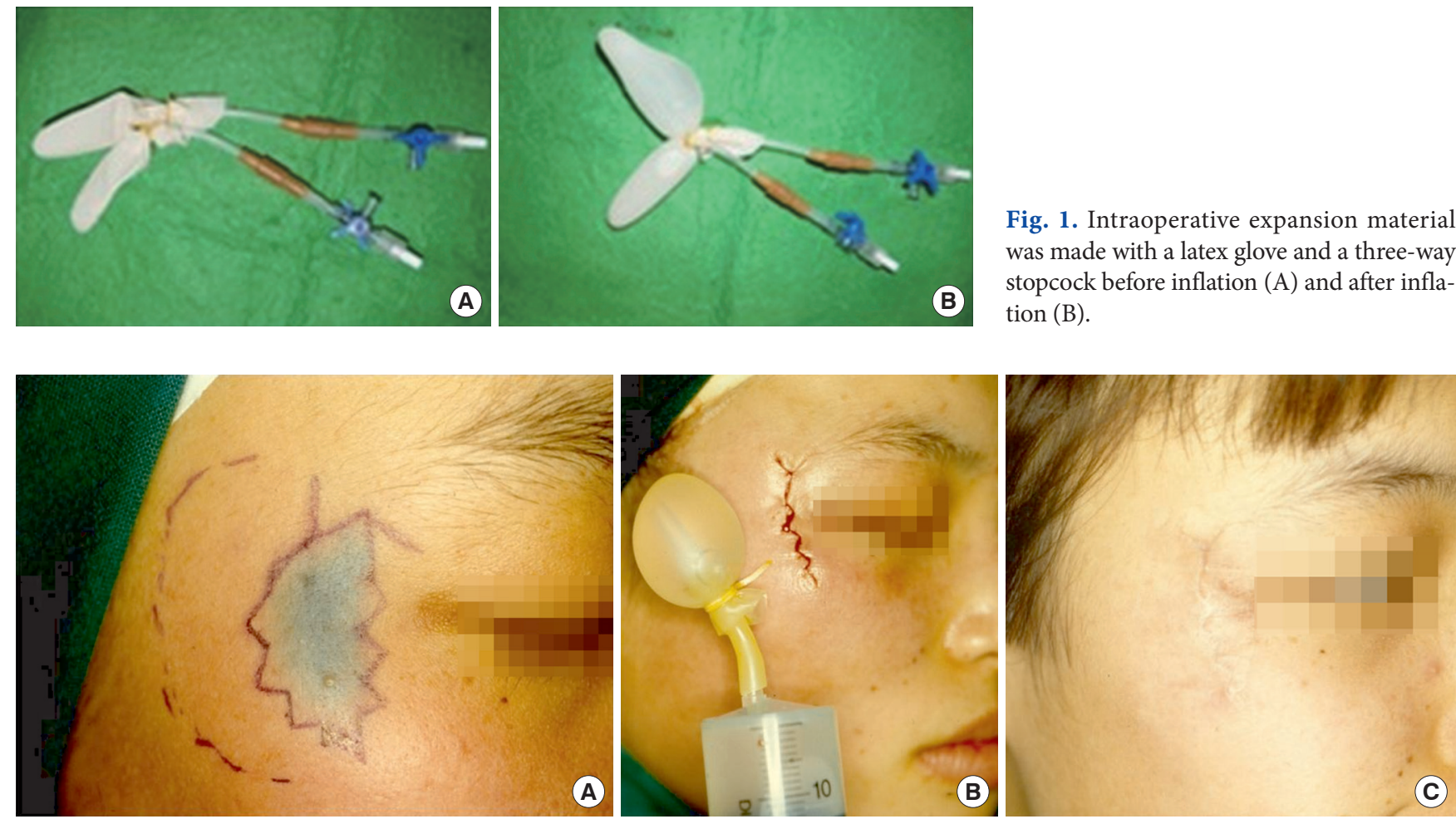

Fig. 1. Intraoperative expansion material was made with a latex glove and a three-way stopcock before inflation (A) and after inflation (B).

Fig. 2. A blue nevus at the temple area (A) was excised, after insertion of intraoperative expansion (B), and coverage was done with minimal scarring $(\mathrm{C})$.
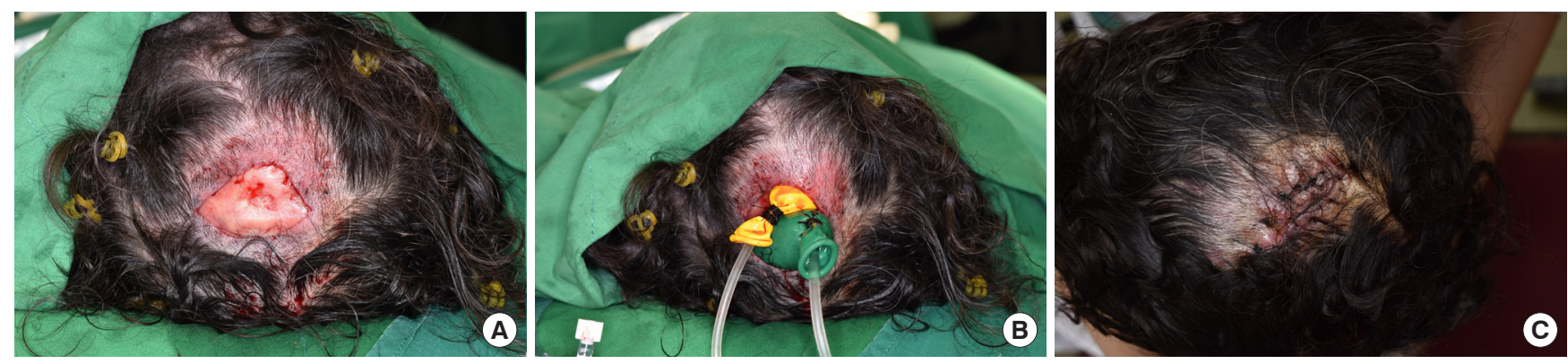

Fig. 3. A $5 \times 5-\mathrm{cm}$ skin and soft tissue defect in the parietal area after mass excision (A), scene of balloon inflation (B), and the wound site after the surgery showing approximation with minimal tension $(\mathrm{C})$.

loons) and the location of the expander (either unilateral or bilateral placement under the incision site). Finally, to prevent dehydration in the expanded tissue and to boost healing, sufficient amount of antibacterial lubricant was applied for 3 to 5 days.

There were no early or late postoperative complications such as contracture of the skin, wound dehiscence, inflammation, infection, hematoma or fluid collection. Slight ecchymoses were observed, which was spontaneously resolved within 3 weeks. Tension was minimal; texture was similar to that of adjacent tissue. Scarring was also minimized, leaving a fine linear scar by postoperative scar management which included taping, silicone sheets and ointment (Figs. 2, 3). The average expansion diame- 
and pregnancy, as well as the expansion of skin covering tumors. This has been accepted as a new form of tissue expansion since Neumann [2] first used a tissue expander to treat microtia. Expanding skin tissue using expanding devices were further developed by Radovan [3] and Austad and Rose [4], who used balloons. This was followed by the introduction of ISLE by Sasaki [1], as mentioned earlier. The physical properties of skin used in tissue expansion are viscoelasticity, tension and distensibility; these properties largely depend on the fibrous weave of the dermis. Tissue expansion occurs as a result of two phenomena-creeping and stress relaxation-in which the viscoelasticity of the skin is changed via the exertion of mechanical pressure. Creeping is a phenomenon in which the skin is stretched by a constant stretching force: the skin is continuously stretched further by the force exerted. Stress relaxation is a phenomenon in which the force required to stretch the skin by a given distance gradually grows smaller [5].

While excessive undermining for primary closure can exhibit complications such as dehiscence or marginal necrosis, some authors argue that intraoperative expansion does not improve tissue gain over undermining, but in clinically other articles suggest additional tissue gain does exist, which was also observed in this study. Further research is necessary to clarify the exact mechanism [6-9].

Skin needling, approximation with towel clips or skin hooks are widely used for rapid tissue expansion to release tension. However, these methods inherently cause trauma to the skin and even lacerations in areas with high tension. Moreover, key structures are damaged during needling or in other methods of expansion. This method has superiority for not causing any trauma to the external skin and preserving key anatomic structures.

The traditionally used tissue expander had several disadvantages, such as which patients had to undergo several surgeries and high expense [10]. In other hands, cheap and easily procured latex gloves or balloons can be expanded up to 300-400 $\mathrm{mL}$ using three digits of a latex glove, while one digit can be expanded up to $30-50 \mathrm{~mL}$ on average and two digits can be expanded up to $200 \mathrm{~mL}$. In addition, one pair of latex gloves can be expanded up to $600 \mathrm{~mL}$, allowing advanced options to various sizes of defects both economically and effectively.

Possible complications include sensory abnormalities, flap loss, dehiscence, infection and hyperpigmentation, which were not observed in our cases. Safety issues can be raised: latex gloves can leave powders under the defect and balloons were simple products from convenient stores. However, the contact time between these materials and the defect was short (always less than an hour) and massive irrigation to rinse off the possi- ble residual materials was performed. There was no single case regarding toxicity in our cases.

The major advantages of intraoperative expansion include fewer requirements for facilities, devices, reduced days of hospitalization and faster recovery after surgery. In addition, this technique is inexpensive, can be produced depending on the defect size, and leaves fine linear scar without distortion and deformity of the surrounding key structure.

The limitation of the intraoperative expansion are as follows: first, although tissue closure could be achieved through one stage, operation time was prolonged in the inflation and deflation processes. Second, as temporary expansion, there was limited amount of expansion than conventional tissue expanders. Continued research on the biomechanics of rapid tissue expansion, as well as postoperative observations, will be needed so as to expand the practice of intraoperative expansion and develop more indications, especially for large defects.

\section{CONFLICT OF INTEREST}

No potential conflict of interest relevant to this article was reported.

\section{PATIENT CONSENT}

The patients provided written informed consent for the publication and the use of their images.

\section{REFERENCES}

1. Sasaki GH. Intraoperative sustained limited expansion (ISLE) as an immediate reconstructive technique. Clin Plast Surg 1987;14:563-73.

2. Neumann CG. The expansion of an area of skin by progressive distention of a subcutaneous balloon; use of the method for securing skin for subtotal reconstruction of the ear. Plast Reconstr Surg (1946) 1957;19:124-30.

3. Radovan C. Tissue expansion in soft-tissue reconstruction. Plast Reconstr Surg 1984;74:482-92.

4. Austad ED, Rose GL. A self-inflating tissue expander. Plast Reconstr Surg 1982;70:588-94.

5. Gibson T. Physical properties of skin. J Anat 1969;105(Pt 1): 183-4.

6. Siegert R, Weerda H, Hoffmann S, Mohadjer C. Clinical and experimental evaluation of intermittent intraoperative shortterm expansion. Plast Reconstr Surg 1993;92:248-54.

7. Wee SS, Logan SE, Mustoe TA. Continuous versus intraoperative expansion in the pig model. Plast Reconstr Surg 1992;90: 
808-14.

8. Sasaki GH. Tissue expansion. West J Med 1989;150:77-8.

9. Lam AC, Nguyen QH, Tahery DP, Cohen BH, Sasaki GH, Moy RL. Decrease in skin-closing tension intraoperatively with suture tension adjustment reel, balloon expansion, and under- mining. J Dermatol Surg Oncol 1994;20:368-71.

10. Demirseren ME, Ceran C, Demirseren DD. Treatment of a congenital melanocytic nevus on the forehead with immediate tissue expansion technique: a three-year follow-up. Pediatr Dermatol 2012;29:621-4. 